\title{
THE ORIENTED HOMOTOPY TYPE OF 3-MANIFOLDS
}

\author{
BY C. B. THOMAS
}

Communicated by W. S. Massey, November 9, 1965.

In [3] Milnor shows that given a connected, closed, compact and oriented 3-manifold $M$, there is up to order a unique expression of $M$ as the connected sum of prime manifolds. The purpose of this note is to sketch how this result can be used to define an invariant of oriented homotopy type for the manifold $M$. A detailed exposition will appear elsewhere.

Throughout a map is assumed to be base point preserving, and an oriented homotopy equivalence between $M_{1}$ and $M_{2}$ is written $M_{1} \simeq_{\circ} M_{2}$.

If one neglects any counterexamples to the Poincaré Conjecture, prime manifolds are of three kinds: handles or copies of $S^{2} \times S^{1}$, aspherical spaces and quotients of finite group actions on homotopy spheres. Examples of the second kind are bundles over $S^{1}$ fibered by 2 -manifolds, and of the third ordinary lens spaces $L(p, q)$. These are classified up to homotopy type by the fundamental group in case two, and by the fundamental group plus the first $k$-invariant from a Postnikov system in case three. In fact we have the following theorems, generalized from 3 to any odd dimension.

Theorem 1. Let $n$ be odd and $Y$ an $n$-dimensional $C W$-complex with finite fundamental group of order $r$ and universal cover $\tilde{Y}$ homotopically equivalent to $S^{n}$. Suppose that $Y$ is given a definite orientation so that $k^{n+1}$ belonging to $H^{n+1}\left(\pi_{1}(Y, y), Z\right)$ is unambiguously defined. Then the Bockstein map describes a natural isomorphism

$$
\beta_{r}: H^{n}\left(\pi_{1}(Y, y), Z_{r}\right) \cong H^{n+1}\left(\pi_{1}(Y, y), Z\right),
$$

and $\beta_{r}^{-1} k^{n+1}$ is mapped onto the orientation class of $Y$ modulo $r$ under the inclusion of $Y$ in the Eilenberg-MacLane complex $K\left(\pi_{1}(Y, y), 1\right)$ obtained by adding cells.

Theorem 2. Given two such complexes $Y_{1}$ and $Y_{2}$, then $Y_{1} \simeq_{\mathrm{O}} Y_{2}$ if and only if there is an isomorphism

$$
\phi: \pi_{1}\left(Y_{1}, y_{1}\right) \stackrel{\sim}{\longrightarrow} \pi_{1}\left(Y_{2}, y_{2}\right)
$$

such that $\phi^{*} k_{2}=k_{1}$.

To prove Theorem 1 one looks carefully at the constructions of 
$k^{n+1}$ and $\beta_{r}$. Sufficiency in Theorem 2 comes from the construction of a map $f: Y_{1} \rightarrow Y_{2}$ realizing $\phi$; the condition on the $k$-invariants ensures that $f$ has the correct degree for homotopy equivalence. Necessity is a consequence of the naturality of the chosen Postnikov construction, see [1].

Now let $M$ be any connected, compact, closed and oriented 3manifold. Its decomposition into primes presents $\pi_{1}(M, m)$ as an irreducible free product $\pi_{1}(M, m)=F * G_{1} * \cdots G_{s} * \cdots G_{t}$, where $G_{1}, \cdots, G_{s}$ are finite of orders $r_{1}, \cdots, r_{s}, G_{s+1}, \cdots, G_{t}$ are infinite and $F$ is free of rank $h$. Write $r$ for the product $2 r_{1} r_{2} \cdots r_{s}$. In dimensions greater than one the cohomology of $\pi_{1}(M, m)$ breaks up into the direct sum of the cohomologies of its factors, and for any prime manifold of the third kind the first $k$-invariant lies in $H^{4}\left(G_{i}, Z\right)$. As in Theorem 1 the Bockstein $\beta_{r}$ (different $r$ ) gives a natural isomorphism between this group and $H^{3}\left(G_{i}, Z_{r}\right)$, we define $q_{i}=\beta_{r}^{-1} k_{i}^{4}$, $i=1,2, \cdots, s$. For the aspherical prime manifolds define $q_{i}$ to be the orientation classes modulo $r$ in $H^{3}\left(G_{i}, Z_{r}\right), i=s+1, \cdots, t$. Set $q(M)=\left\{q_{1}, \cdots q_{*} \cdots, q_{\imath}\right\}$ in $H^{3}\left(\pi_{1}(M, m), Z_{r}\right)$.

Theorem 3. Two manifolds $M_{1}$ and $M_{2}$ have the same oriented homotopy type if and only if there is an isomorphism

$$
\phi: \pi_{1}\left(M_{1}, m_{1}\right) \stackrel{\sim}{\rightrightarrows} \pi_{1}\left(M_{2}, m_{2}\right)
$$

such that $\phi^{*} q\left(M_{2}\right)=q\left(M_{1}\right)$.

Notes. By a result on irreducible free products, see [2], $\phi$ can be chosen to map $G_{i}\left(M_{1}\right)$ onto $G_{i}\left(M_{2}\right)$ for each $i$.

The factor 2 in the definition of $r$ is needed to distinguish orientations in the case when both $M_{1}$ and $M_{2}$ are the connected sums of projective space with an aspherical manifold.

The handles give no trouble since by Theorem 32.1 in [4] they are completely determined by the free group $F$ in the presentation of $\pi_{1}(M, m)$.

\section{REFERENCES}

1. D. W. Kahn, Induced maps for Postnikov systems, Trans. Amer. Math. Soc. 107 (1963), 432-450.

2. A. G. Kurosh, Theory of groups, Vol. II, Chelsea, New York, 1956.

3. J. Milnor, A unique decomposition theorem for 3-manifolds, Amer. J. Math. 84 (1962), 1-6.

4. C. D. Papakyriakopoulos, Dehn's lemma and the asphericity of knots, Ann. of Math. 66 (1957), 1-26.

Trinity College, Cambridge University and CORNELl UNIVERSITY 